Cuestiones de Sociología, no 18 , e052, junio 2018, ISSN 2346-8904 Universidad Nacional de La Plata Facultad de Humanidades y Ciencias de la Educación Departamento de Sociología

\title{
Notas sobre la relación entre Política Pública y Deporte en la Argentina contemporánea (2008-2018)
}

\author{
Levoratti, Alejo \\ Universidad Nacional de La Plata, Argentina \\ levoratti@gmail.com \\ Moreira, Verónica \\ Instituto de Investigaciones Gino Germani - Universidad de \\ Buenos Aires - CONICET, Argentina \\ veromoreira175@gmail.com
}

Cita sugerida: Levoratti, A. y Moreira, V.(2018). Notas sobre la relación entre Política Pública y Deporte en la Argentina contemporánea (2008-2018). Cuestiones de Sociología, 18, e052. https://doi.org/10.24215/23468904e052 


\title{
Notas sobre la relación entre Política Pública y Deporte en la Argentina contemporánea (2008-2018)
}

Notes about the relationship between Public Policy and Sport in contemporary Argentina (2008-2018)

\author{
Alejo Levoratti \\ Universidad Nacional de La Plata, Argentina \\ levoratti@gmail.com \\ Verónica Moreira \\ Instituto de Investigaciones Gino Germani - Universidad de \\ Buenos Aires - CONICET, Argentina \\ veromoreira175@gmail.com
}

\section{RESUMEN:}

El presente trabajo tiene como objetivo mostrar algunas de las manifestaciones que adopta la relación entre Estado y Deporte en la historia reciente de Argentina. En particular, el trabajo busca problematizar la inscripción del deporte en las agencias estatales nacionales y el lugar que estas últimas han desempeñado en la dirección de las políticas deportivas, considerando principalmente -aunque no de manera exclusiva- los últimos diez años (2008-2018).

Palabras Clave: Políticas Públicas, Deporte, Agencias estatales, Múltiples significados.

\section{Abstract:}

This paper's purpose is to show some of the manifestations that the relation between State and Sport adopted in the recent Argentinian history. Particularly, the work attemp to problematize the registration of sport in national organizations and the place in which they work in the direction of sports policies, taking into account (although not exclusively) the last ten years (2008-2018).

Keywords: Public Policies, Sports, State Agencies, Multiple meanings.

\section{INTRODUCCIÓN}

El presente trabajo tiene por objeto producir una serie de reflexiones a partir de las investigaciones realizadas por los autores tanto en instancias previas sobre las políticas públicas deportivas en diferentes agencias estatales (Levoratti, 2015 y 2017; Moreira, 2015) como así también en las labores actuales en el marco del grupo de trabajo de CLACSO Deporte, Políticas Públicas y Sociedad. A partir de ello se busca problematizar la inscripción del deporte en las agencias estatales en Argentina y el papel que estas últimas han desempeñado en la dirección de las políticas deportivas, considerando principalmente -aunque no de manera exclusivalos últimos diez años (2008-2018). Así, el presente estudio focaliza la mirada en la relación entre Estado y deporte en la Argentina contemporánea.

Hacia fines de 2007 asume la presidencia de la nación Cristina Fernández de Kirchner, momento en el cual la Secretaría de Deportes, que dependía de Presidencia de la Nación, es transferida al Ministerio de Desarrollo Social. Este acontecimiento implicó una serie de cambios en la inscripción del deporte dentro del Estado. Durante este mandato presidencial se sancionaron distintas normativas en torno a las prácticas deportivas ${ }^{1}$. Como corolario del proceso, a fines de octubre de 2015 se sancionaron las leyes No 27201 y $27.202^{2}$, tendientes a legalizar nuevas modalidades de gobierno del deporte, así como también a visibilizar otras maneras de significar y valorar dicho fenómeno ${ }^{3}$. Con la asunción del Ingeniero Mauricio Macri como primer mandatario de Argentina y de Carlos Javier Mac Allister como Secretario de Deportes de la Nación en diciembre de 2015, los lineamientos del marco normativo impulsados hasta ese momento comenzaron 
a tensionarse con los cambios ideados por el gobierno entrante. La medida más representativa del último gobierno en relación con este tema fue el freno a la implementación de las dos leyes mencionadas líneas arriba.

Por lo expuesto, resulta interesante identificar en este breve recorrido las modalidades y los significados que ha adquirido la inserción del deporte en las agencias estatales. En este recorrido, se presentarán algunos puntos nodales de las políticas deportivas del período seleccionado.

\section{EsTAdo y DePORTE, UNA RELACIÓN DINÁMICA Y CON HISTORIA}

Este apartado busca graficar cómo en algunos períodos del siglo XX la problemática del deporte se fue inscribiendo en simultáneo en distintas agencias estatales, donde se posicionaron actores y grupos que en paralelo lo hacían en otros espacios sociales. Bohoslavsky y Soprano (2010) exponen la necesidad de considerar al Estado como un espacio polifónico en el que se expresan diferentes grupos sociales, donde diversos agentes estatales producen y actualizan sus representaciones y prácticas cotidianas en interlocución con individuos inscriptos en sectores formalmente exteriores al estatal, pero que inciden en la configuración de las representaciones y prácticas de los primeros. Se pueden identificar a lo largo del siglo XX en el Estado Nacional distintas agencias estatales, donde se inscribieron grupos sociales singulares que se encargaron de direccionar y promover sus concepciones y propuestas acerca de las prácticas deportivas.

En este sentido, parece significativo mencionar que, hacia fines Siglo XIX e inicios de la siguiente centuria, dentro del Ejército Argentino se dio la participación de ciertos actores sociales que promovieron y conformaron la Escuela Militar de Gimnasia y Esgrima. Entre ellos se destacaron: el Barón Antonio de Marchi, Marcelo T. de Alvear, Carlos Pellegrini y Eugenio Pini, quienes impulsaron las prácticas gímnicas $\mathrm{y}$, en ámbitos particulares, las prácticas deportivas como la esgrima. Dichos actores se vinculaban a la elite porteña y a dirigentes políticos que participaban asiduamente en el Club Gimnasia y Esgrima de Buenos Aires, el Jockey Club, el Círculo de Armas, la Sociedad Sportiva. Por su parte, algunos maestros de gimnasia y esgrima del Ejército fueron funcionarios de administraciones estatales tales como las Direcciones Municipales. Este fue el caso de Arsemio Thamier, que ejerció funciones en la Dirección de Plazas Públicas de la por entonces Capital Federal, o de Juan Arrospidegaray como Director del Stadium Municipal e Inspector de Plazas y Campos de Deportes de la Municipalidad de Rosario (Roldán, 2012). También, cabe destacar que, simultáneamente, en el Ministerio de Instrucción Pública del Estado Nacional se promovían otros sentidos sobre las prácticas corporales, direccionados preponderantemente bajo la figura del Doctor Enrique Romero Brest, donde inicialmente las prácticas deportivas se encontraban subsumidas bajo las prácticas gímnicas (Scharagrosky, 2014; Levoratti, 2015b).

En esa dirección, Eduardo Archetti (2001) considera que hacia inicios del siglo XX los promotores de la gimnasia lograron inscribirse hacia dentro del Estado, mientras que "la expansión del deporte en la Argentina se puede asociar al desarrollo de la sociedad civil ya que las organizaciones y clubes deportivos generaron espacio de autonomía y participación social al margen del Estado" (Archetti, 2001, p. 12) ${ }^{4}$. Este autor identifica en el período de diez años de gobierno peronista (1945-1955) el momento donde se promovió de modo regular desde el Estado una política sistemática y direccionada hacia la relación entre deporte y nación.

El deporte se inscribió de múltiples modos en distintas dependencias del Estado. Se puede ver este proceso en el siguiente ejemplo. En abril de 1963, sobre la base de la Dirección General de Educación Física, se creó en el Ministerio de Educación y Justicia la Dirección Nacional de Educación Física y Recreación, la cual tuvo competencias orientadas a la organización y promoción de la Educación Física y el deporte preponderantemente en el ámbito educativo "formal". Esta agencia estatal es cerrada en el año 1993, cuando en el marco de las reformas educativas de los años 90 se promueve una nueva organización del Ministerio de Educación de la Nación.

La Ley 20.655 de Promoción de las Actividades Deportivas fue sancionada el 21 de marzo de 1974 durante el tercer mandato presidencial de Juan Domingo Perón. Esta reglamentación sustituyó a la Ley 18.247 
de Fomento y Desarrollo del Deporte, sancionada en 1969 durante el gobierno de facto de Juan Carlos Onganía. En el artículo primero de la Ley se pueden identificar algunos de los sentidos asociados al deporte en tanto objeto polisémico como "factor educativo coadyuvante a la formación integral del hombre y como recurso para la recreación y esparcimiento de la población (...) como un factor de la salud física y moral de la población", procurando que las "representaciones del deporte argentino a nivel internacional sean la real expresión de la jerarquía cultural y deportiva del país” (Ley 20655 de 1974). Además, se le asignó al Estado la responsabilidad de desarrollar una acción "orientadora, promoviendo, asistiendo, ordenando y fiscalizando las actividades deportivas del país”, procurando así un carácter federal. Para ello se estableció la creación de un área específica para la temática dentro del Ministerio de Bienestar Social, así como la organización del Fondo Nacional del Deporte y el Consejo Nacional del Deporte que se encargaron de asesorar al organismo rector. Dicho organismo estatal (la cartera dedicada al deporte) durante los distintos gobiernos democráticos modificó su estatus y su inscripción dentro del Estado, siendo considerada una dirección de estado, una subsecretaria y una secretaría de estado (máximo rango alcanzado), al mismo tiempo que dependió del Ministerio de Bienestar Social, de la Secretaría de Presidencia de la Nación, del Ministerio de Turismo y Deporte, del Ministerio de Desarrollo Social y más recientemente del Ministerio de Educación y Deporte ${ }^{5}$.

Estos cambios implicaron modificaciones en los recursos económicos dedicados del deporte, así como también modificaciones en las relaciones de dependencia o supeditación de este en distintas agencias estatales. Tales cambios fueron acompañados, en muchos casos, por diversas configuraciones de la práctica deportiva.

\section{DePorte, POLÍticas PÚBLICAS y AgenCias ESTATALES (2008-2018)}

En diciembre de 2007 tomó posesión como presidenta de Argentina Cristina Fernández de Kirchner. A partir de su asunción, la Secretaría de Deportes, que se encontraba bajo la dependencia de la Secretaría de la Presidencia de la Nación, fue transferida a la órbita del Ministerio de Desarrollo Social de la Nación, continuando a cargo de dicha dependencia Claudio Morresi ${ }^{6}$. Haciendo una evocación sobre esta incorporación, la Ministra de la mencionada cartera, Alicia Kirchner, se refirió a este cambio en los siguientes términos: “...con visión de futuro y con gran esperanza...” dado “... el reconocimiento y valor potencial e instrumental que le reconocemos a la actividad física, el deporte y la recreación para el desarrollo humano". Esta gestión elaboró el "Plan estratégico del deporte argentino 2008-2012" donde se manifestó que el desarrollo del deporte es una responsabilidad del Estado, pues concibe que existe un "Derecho al deporte y a la Actividad Física" que es de "todos y todas". Con el plan se buscó producir una consolidación de una “... cultura de la actividad física y el deporte, que aprende con otros y de otros procurando un fin: la inclusión, integración, equidad y búsqueda de la calidad como requisito para el desarrollo nacional”. También, en el marco teórico del "Plan estratégico del deporte argentino 2008-2012" se encontraron algunos de los sentidos desplegados en la "Ley del Deporte No 20.655", que rescataba el carácter polisémico del término: "El deporte no tiene valores en sí mismo, sino que se construyen por juicios subjetivos que emiten las personas que lo planifican, lo practican, lo conducen o sobre la base de los efectos que éstos creen obtener" (Argentina, 2008, p. 10). Dentro de esta caracterización, la Secretaría de Deportes de la Nación incorporó una clasificación según las siguientes categorías:

- Deporte social “es la práctica de Actividad Física y Deportivas orientadas a la población en su conjunto, sin discriminación de edad, sexo, condición física, social, cultural o étnica, (...) generadora de situaciones de inclusión...” (Argentina, 2008, p. 11. Destacado nuestro);

- Desarrollo deportivo: “... Es un proceso en el que los deportistas pueden incluirse, participar y continuar con posibilidades de éxito el tránsito hacia el camino al rendimiento deportivo" (Argentina, 2008, p. 11); y por último 
- Deporte de Representación Nacional: “...actividad deportiva organizada, correspondiente al campo del Deporte Federado y de seleccionados nacionales...”(Argentina, 2008, p. 11).

Cabe destacar que cada una de estas clasificaciones contó con un plan nacional, en donde se incorporaron una serie de programas y proyectos que implementó la Secretaría.

En el año 2009, a instancias del presidente del Comité Olímpico Argentino ${ }^{7}$, Gerardo Werthein ${ }^{8}$, fue sancionada la Ley 26.573 a partir de la cual se creó el Ente Nacional de Alto Rendimiento Deportivo (ENARD). Este organismo, de acuerdo al marco legal, se inscribió como una persona jurídica de derecho público no estatal, compartiéndose a partir de ese momento la dirección de las políticas deportivas de alto rendimiento entre el Comité Olímpico Argentino y la Secretaria de Deportes de la Nación. Actualmente, el Directorio está formado por representantes de la Secretaría de Deportes de la Nación, el Comité Olímpico y dos exatletas de olímpicos o de alto rendimiento. Entre las funciones principales del ENARD, se encontraban las de gestionar y coordinar el apoyo económico para los atletas y entrenadores, contemplando distintos niveles de representación nacional; brindar becas a deportistas amateurs; completar los subsidios de la Secretaría de Deportes de La Nación para competencias internacionales. Un punto de la ley, que generó polémica durante la gestión del presidente Mauricio Macri, fue el destinado al financiamiento del organismo. El ENARD logró funcionar desde su inicio con el financiamiento a partir del cargo del uno por ciento aplicado al precio del abono que las empresas de telefonía celular facturaban -y siguen facturando-a sus clientes.

Precisamente, en el año 2017, en el marco de la reforma tributaria impulsada por el Ministerio de Hacienda de la Nación, se buscó suprimir el impuesto a la telefonía celular por el cual se financiaba el ENARD. Esto llevó a que las autoridades del Comité Olímpico Argentino y deportistas realizaran campañas de protesta principalmente en medios periodísticos y en las redes sociales, ocasionando que dicho ministerio incluyera fondos en el presupuesto nacional para el ENARD. Dicha medida puede considerarse como una pérdida de autonomía por parte del ente en relación al poder ejecutivo, dado que su partida presupuestaria queda supeditada al presupuesto nacional.

Cabe destacar que, en razón del gobierno anterior, hacia el año 2015 el tratamiento del deporte recibió un impulso en pos de su crecimiento y desarrollo a partir de la participación de distintos sectores sociales en el foro Conectados x Deporte; un espacio de reunión periódica de alcance federal que fue promovido por diputados del Frente para la Victoria pertenecientes a las Comisiones de Deportes, Salud y Educación, entre los que se destacó el diputado nacional Mauricio Gómez Bull. En dicho ámbito tuvo lugar el intercambio que dio paso al borrador de las leyes 27.201 y 27.202, sancionadas y promulgadas hacia fines de $2015^{9}$,y que el presidente actual dejó sin efecto.

La ley 27.201 impartió la creación del Ente Nacional de Desarrollo Deportivo (ENADED), entendido:

Como persona jurídica de derecho público no estatal destinado a gestionar y coordinar: en lo nacional, una estructura de administración, coordinación y apoyo al deporte y la actividad física; en lo provincial, concretar una armónica realización de esfuerzos tendientes al logro de tal estructura; en lo municipal, apoyar la satisfacción y necesidades primarias a través de las estructuras municipales deportivas reconocidas en la ley 20.655.(Ley $\mathrm{N}^{\circ} 27201 ; 2015$ )

Es decir, un organismo capaz de implementar planes, programas, proyectos y acciones nacionales, provinciales y municipales en pos del desarrollo de infraestructura deportiva en centros de mediano y alto rendimiento y de clubes federados y barriales. En este contexto, un punto novedoso de la ley fue la Asignación Universal por hijo en el deporte, que planteaba darle un suplemento adicional a cada persona menor de edad o persona con discapacidad, entre los seis y dieciséis años de edad, que estuvieran a cargo de titulares de derecho que percibían la Asignación Universal por Hijo para Protección Social. El ente sería administrado por una dirección ejecutiva compuesta por doce integrantes: seis representantes del Observatorio Nacional del Deporte y la Actividad Física y seis representantes de la Confederación Argentina de Deportes. Esta 
conformación pretendía descentrar de las agencias de estado las definiciones de los lineamientos de las políticas deportivas nacionales, al mismo tiempo que daba participación a las confederaciones y deportistas.

La perspectiva de género fue un aspecto innovador de la ley 27202, que propuso la igualdad de oportunidades para las mujeres de participar e intervenir en todos los niveles de adopción de decisiones en el deporte y la actividad física. Entre los puntos centrales de la misma se destaca:

La creación de un Programa Nacional de Empoderamiento de la Mujer en el Deporte, que tendrá como finalidad garantizar la igualdad, participación, inclusión, acceso y representación de las mujeres en todos los ámbitos y a todos los niveles de la comunidad deportiva, tales como: atletas, practicantes, gestoras, dirigentes, entrenadoras, técnicas, árbitras, juezas...(Ley $\mathrm{N}$ $\left.{ }^{\circ} 27202 ; 2015\right)$

Dicho proceso se llevaría a cabo a partir de acciones tales como cursos, debates, foros, seminarios y talleres de capacitación que posibiliten: a) el acceso equitativo de la mujer en el deporte; b) asegurarla formación con perspectiva de género de profesionales del deporte en los ámbitos federativos; c) promoverla equidad en formatos competitivos, distribución geográfica, visibilidad y recompensas de los deportes de competición, y d) concientizar y postular un trabajo de construcción colectiva en el ámbito de las federaciones deportivas, como posibles vehiculizadores de situaciones de violencia familiar o abuso sexual infantil en atención a la inmediatez y desarrollo del trabajo formativo que el deporte supone en niñas, niños y adolescentes.

\section{FINAL}

A partir de la breve descripción de casos recientes, este trabajo expone las diferentes modalidades de inscripción del deporte en las agencias del Estado Nacional. En ese recorrido se puede advertir la presencia de múltiples agencias donde actores y grupos sociales particulares, representativos del Estado o de espacios ajenos a este, pregonan múltiples sentidos sobre las prácticas deportivas, y también la posición del Estado para el desarrollo y la promoción del deporte en sus diferentes variantes (deporte recreativo, alto rendimiento, intermedio entre ambas categorías). Este proceso de imbricación de agencias y participación de actores originarios de espacios distintos se ha dado, como se mostró, a lo largo de la historia reciente en Argentina. A partir de algunos pasajes de las leyes sancionadas se han podido observar, además, los valores que el deporte como categoría polisémica conlleva: desde las consecuencias directas para la salud, la centralidad para el esparcimiento y la recreación, la búsqueda de la integridad moral, hasta la importancia como fenómeno representativo de lo nacional a través del desarrollo de las disciplinas deportivas tendientes al alto rendimiento.

Otro aspecto singular del período es la articulación que se estableció entre las agencias estatales con organismos, asociaciones y grupos sociales (tales como atletas, técnicos y dirigentes) para la definición y el desarrollo de las líneas de acción. Este proceso se observó con claridad con la creación en 2009 del ENARD, y en 2015 del ENADED (sin vigencia).

A partir de este breve diagnóstico surgen tres líneas de investigación.

Un punto que resulta necesario analizar son los procesos históricos de configuración de las políticas deportivas en la Argentina, identificando en el devenir histórico a las instituciones, proyectos y actores. Esto nos posibilita estudiar en profundidad el lugar de las agencias estatales en la definición de las políticas públicas, como así también las vinculaciones con otros espacios sociales por fuera del estatal.

Por otro lado, continuar con la indagación sobre las políticas públicas deportivas del gobierno actual. En particular, identificar cuáles son los lineamientos normativos establecidos durante el gobierno de Cristina Fernández de Kirchner que continúan en ejecución y los que fueron eliminados, y las nuevas líneas de la política pública promovidas desde la gestión de la coalición Cambiemos. En este contexto, sigue siendo un tema de interés reflexionar sobre las trayectorias de los y las atletas, los recursos públicos y/o privados que 
ayudan para el financiamiento de sus carreras, los mecanismos de exclusión-inclusión de los atletas para el alto rendimiento y los procesos de mercantilización de sus figuras (puntos centrales del proyecto CLACSO).

En dicho contexto, otro aspecto es identificar cuál ha sido y cuál es el rol actual de las mujeres en el deporte. Esta línea de investigación no sólo se desprende de la decisión del gobierno actual de no aplicar las leyes sancionadas y promulgadas hacia fines de 2015, ambas con perspectiva de género, sino también de un proyecto en etapa de evaluación a partir del cual buscamos reflexionar sobre las dificultades de acceso de las mujeres en el deporte. En este sentido, es primordial identificar en la vida contemporánea la pervivencia de barreras de orden cultural, la distribución desigual de los recursos económicos, de oportunidades sociales que tienen las mujeres actualmente; pensar así en la igualdad de oportunidades en términos de género de participar e intervenir en todos los niveles de adopción de decisiones y prácticas en el deporte y la actividad física. La elección del tercer eje cobra relevancia en nuestra coyuntura particular: las discusiones sobre la inclusión de las mujeres en distintos ámbitos de la sociedad contemporánea y el movimiento de mujeres por la igualdad de derechos que se expande con fuerza en distintas ciudades del país.

\section{Bibliografía}

Archetti, E. (2001). El potrero, la pista y el ring. Las patrias del deporte argentino. Buenos Aires: Fondo de Cultura Económica.

Argentina, Ministerio de Desarrollo Social de la Nación, Secretaría de deportes de la Nación. (2008). Plan estratégico de deporte Argentino 2008-2012. Recuperado de:http://deportes.gov.ar/ar/site/planestrategico/

Daskal, R. (2017). Deporte y política: apuntes para una discusión. En Ron, O.et al.,Actas del primer encuentro deporte $y$ sociedad. La Plata: FaHCE.

Daskal, R. y Moreira, V. (2017).Clubes argentinos. Debates sobre un modelo. Buenos Aires: UNSAM edita.

Levoratti, A. (2015). Deporte y politica socio-educativa. Una etnografía sobre funcionarios y profesores de educación fisica. Buenos Aires: Prometeo Libros.

Levoratti, A. (2015b). El "deporte" en la formación de los "profesores normales de Educación Física" en Argentina (1912-1940). Revista Teoria e Prática da Educação, 18(1), 135-145.

Levoratti, A. (2017). El deporte en el Estado nacional: continuidades y rupturas (Argentina, 1989-2015). Materiales para la Historia del Deporte, 15.

Ley No 20.655 (1974). Ley del deporte.

Ley No 26.573 (2009). Ente Nacional de Alto Rendimiento Deportivo (ENARD).

Ley No 27.201 (2015). Ente Nacional de Desarrollo Deportivo (ENADED).

Ley No 27.202 (2015). Ley del deporte.

Moreira, V. (2015). Deporte y política pública en el municipio de avellaneda: Articulaciones entre lo local y lo nacional. Revista Lúdica Pedagógica, 21, 53-61.

Roldán, D. (2012).La invención de las masas. Ciudad, corporalidades y culturas. Rosario, 1910- 1945. La Plata: Universidad Nacional de la Plata.

Scharagrosky, P. (2014). El discurso médico y su relación con la invención del oficio de 'educador físico': entre la heteronomía solapada y la autonomía vigilada (Argentina 1901 -1931). En Scharagrodsky, P. (Compilador), Miradas Médicas sobre la cultura física en Argentina 1880-1970. Ciudad Autónoma de Buenos Aires: Prometeo Libros.

\section{Notas}

1 Durante este período se sancionaron las siguientes leyes: Ley No 26.462 (2008) Juegos Nacionales Evita; Ley No 26.573 (2009) Ente Nacional de Alto Rendimiento Deportivo (ENARD); Ley No 27.098 (2015) Régimen de Promoción de 
los Clubes de Barrio y de Pueblo; Ley No 27.201 (2015) Ente Nacional de Desarrollo Deportivo (ENADED) y la Ley No 27.202 (2015) Ley del deporte.

2 Ley No 27.201 Ente Nacional de Desarrollo Deportivo (ENADED) y la Ley No 27.202 Ley del deporte.

3 Entre los conceptos clave que atravesaron los discursos de los funcionarios se destacan: la inclusión y la igualdad de oportunidades de la práctica deportiva. Por otra parte, dicho período se distingue, como se muestra más adelante, por la creación de un organismo dedicado a la organización del deporte de alto rendimiento.

4 No obstante, dichas instituciones deportivas han recibido a lo largo de su historia ayudas de diferente especie de parte del Estado nacional, provincial y local (cesión de tierras fiscales, subsidios) debido al perfil social, no lucrativo, destinado al beneficio de la comunidad, estableciéndose por esto una relación de reciprocidad entre ambos sectores (Daskal y Moreira, 2017).

5 El lector interesado en las inscripciones dentro del Estado Nacional de la problemática del deporte puede ver (Levoratti, 2017).

6 Morresi: es un ex futbolista y entrenador argentino, con activa participación en los organismos de Derechos Humanos.Se desempeñó como Secretario de deportes de la Nación entre 2004-2014.

7 El Comité Olímpico Argentino es una entidad civil, autárquica, desvinculada por normas estatutarias de todo tipo de influencia económica, política, religiosa y racial.

8 Gerardo Werthein es médico veterinario, pertenece a una familia que posee un importante holding empresarial, se desempeña como CEO de Telecom y fue presidente de la Federación Ecuestre Argentina. Desde el año 2009 hasta la actualidad posee el cargo de Presidente del Comité Olímpico Argentino.

9 Ver Daskal (2017) para ampliar sobre los pormenores del debate. 